OPEN ACCESS

Edited by:

Chiranjib Chakraborty, Galgotias University, India

Reviewed by:

Joel D. A. Tyndall,

University of Otago, New Zealand

Shiqiao Ye,

University of Arkansas for Medical

Sciences, United States

*Correspondence:

Yulong Liang

yulong@bcm.edu

Long Yu

longyu@fudan.edu.cn

tThese authors have contributed equally to this work.

Specialty section:

This article was submitted to Experimental Pharmacology and Drug

Discovery,

a section of the journal

Frontiers in Pharmacology

Received: 16 October 2017

Accepted: 05 February 2018

Published: 21 February 2018

Citation:

Zhao X, Xia C, Wang X, Wang $H$,

Xin M, Yu L and Liang Y (2018) Cyclophilin J PPlase Inhibitors Derived from 2,3-Quinoxaline-6 Amine Exhibit Antitumor Activity.

Front. Pharmacol. 9:126. doi: 10.3389/fphar.2018.00126

\title{
Cyclophilin J PPlase Inhibitors Derived from 2,3-Quinoxaline-6 Amine Exhibit Antitumor Activity
}

\section{Xuemei Zhao ${ }^{1 \dagger}$, Chengcai Xia ${ }^{1 \dagger}$, Xiaodan Wang ${ }^{1}$, Hao Wang ${ }^{1}$, Ming Xin ${ }^{1}$, Long Yu ${ }^{2 \star}$ and Yulong Liang ${ }^{1,3 *}$}

${ }^{1}$ College of Pharmacy, Taishan Medical University, Tai'an, China, ${ }^{2}$ State Key Laboratory of Genetic Engineering, Institute of Genetics, School of Life Sciences, Fudan University, Shanghai, China, ${ }^{3}$ Michael E. DeBakey Department of Surgery, Baylor College of Medicine, Houston, TX, United States

Cyclophilin J (CyPJ), also called peptidylprolyl isomerase like 3, has been identified as a novel member of the cyclophilin family. Our previous research has resolved the three-dimensional structure of CyPJ and demonstrated the peptidylprolyl cis-trans isomerase (PPlase) activity of CyPJ, which can be inhibited by the common immunosuppressive drug cyclosporine A (CsA). Importantly, CyPJ is upregulated in hepatocellular carcinoma (HCC) and promotes tumor growth; CyPJ inhibition by CsA- or siRNA-based knockdown results in a remarkable suppression of HCC. These findings suggest that CyPJ may be a potential therapeutic target for HCC, and discovery of relevant inhibitors may facilitate development of a novel CyPJ-based targeting therapy. However, apart from the common inhibitor CsA, CyPJ has yet to be investigated as a target for cancer therapy. Here, we report structure-based identification of novel small molecule non-peptidic CyPJ inhibitors and their potential as antitumor lead compounds. Based on computer-aided virtual screening, in silico, and subsequently surface plasmon resonance analysis, 19 potential inhibitors of CyPJ were identified and selected for further evaluation of PPlase CyPJ inhibition in vitro. Thirteen out of 19 compounds exhibited notable inhibition against PPlase activity. Among them, the compound ZX-J-19, with a quinoxaline nucleus, showed potential for tumor inhibition; thus, we selected it for further structure-activity optimization. A total of 22 chemical derivatives with 2,3-substituted quinoxaline-6-amine modifications were designed and successfully synthesized. At least 2 out of the 22 derivatives, such as $\mathbf{Z X - J - 1 9 j}$ and $\mathbf{Z X - J - 1 9 I , ~ d e m o n s t r a t e d ~ r e m a r k a b l e ~ i n h i b i t i o n ~ o f ~ t u m o r ~ c e l l ~ g r o w t h , ~ c o m p a r a b l e ~ t o ~}$ CsA but much stronger than 5-fluorouracil. These results indicate that these two small molecules represent novel potential lead compounds for CyPJ-based antitumor drug development.

Keywords: PPIL3, cyclophilin J, quinoxaline derivative, hepatocellular carcinoma, cyclophilin $\mathrm{J}$ inhibitor, peptidylprolyl isomerase, CyPJ, PPlase

\section{INTRODUCTION}

Cyclophilins constitute a superfamily of peptidylprolyl isomerases (PPIase), which catalyze the cis-trans isomerization of peptide bonds on the N-terminal side of proline residue (Harikishore and Yoon, 2015; Dunyak and Gestwicki, 2016). The cyclophilin family, which is comprised of more than 15 members, was named for its ability to bind the common immunosuppressive drug 
cyclosporine A (CsA) (Walsh et al., 1992). Cyclophilins have been shown to act as chaperones accelerating protein folding and maturation, as well as playing a critical role in signal transduction and the immune response (Gothel and Marahiel, 1999; Wang and Heitman, 2005; Lucke and Weiwad, 2011; Harikishore and Yoon, 2015). They have also been implicated in many pathological processes, including viral infection (Towers et al., 2003; Hopkins and Gallay, 2015; Dawar et al., 2017), rheumatoid arthritis (Kim et al., 2005; Pap, 2005), cardiovascular diseases (Satoh et al., 2009; Nigro et al., 2011; Xue et al., 2017), and cancers (Lee and Kim, 2010; Nigro et al., 2013; Wang et al., 2017).

Cyclophilin J (CyPJ), also known as peptidylprolyl isomerase like 3 (PPIL3), is a new member of the cyclophilin family, with human CyPJ being first reported in 2001 (Zhou et al., 2001). CyPJ is encoded by the CyPJ gene initially identified in the fetal human brain. Its protein sequence exhibits $50 \%$ identity to human cyclophilin A and 72\% identity to cyclophilin isoform 10 of Caenorhabditis elegans (Zhou et al., 2001).

Previously, we reported, for the first time, the threedimensional structure of human CyPJ alone and in complex with CsA (Huang et al., 2005). We also identified its PPIase activity, which could be inhibited by CsA (Chen et al., 2015). The CyPJ protein contains four helices and one $\beta$-barrel composed of eight antiparallel $\beta$-strands. It also harbors a disulfide bridge and four segments with conformations strikingly different from those of CyPA (Huang et al., 2005). Specifically, His43, Arg44, and Gln52 are conserved active site residues located in the shallow pocket of CyPJ (Huang et al., 2005). Furthermore, a conserved water molecule is observed close to His43 and Gln52, while Arg44 is involved in hydrogen bonding interactions with CsA, which accounts for the observed inhibitory qualities of CsA on the PPIase activity within CyPJ (Chen et al., 2015). Importantly, we demonstrated that CyPJ is frequently upregulated in a small cohort of hepatocellular carcinoma (HCC), and CyPJ-based targeting leads to tumor suppression of HCC in a xenograft mouse tumor model (Chen et al., 2015). Several studies also report that CyPJ is upregulated in human glioma (Qi et al., 2005), estrogen receptor-negative breast cancer (Couch et al., 2016), and primary gastric adenocarcinoma (Gong et al., 2017), suggesting that the CyPJ enzyme may be an important and potential therapeutic target. However, apart from the common inhibitor of cyclophilins, CsA, CyPJ is yet to be investigated as a molecular target for cancer therapy.

A quinoxaline is an important class of nitrogen-containing heterocycle and this class of compounds plays an important role in drug development due to their potent pharmacological activity (Supplementary Table S1). For instance, marketed drugs, such as Echinomycin, Levomycin, Actinoleutin, Varenicline, Brimonidine, and Quinacillin, all contain a quinoxaline ring. In addition, quinoxaline derivatives have been reported as core motifs in medicinally active ingredients, acting as anti-protozoal, anti-proliferative, anti-inflammatory, anti-bacterial, anti-viral agents, and inhibitors of Jnk stimulatory phosphatase-1 (JSP-1), a special member of the dual-specificity protein phosphatase family 1 (Supplementary Table S1) (Hui et al., 2006; Zhang et al., 2007; Chen et al., 2011). More recently, evidence has emerged that quinoxaline-derived compounds might be capable of inhibiting tumor growth (Supplementary Table S1) (El Aissi et al., 2014; Gu et al., 2017; Miller et al., 2017). However, quinoxaline-based lead compounds have not been evaluated as inhibitors specific for target protein $\mathrm{CyPJ}$ to be applied in cancer therapy.

In this study, we aimed to identify novel potential inhibitors of $\mathrm{CyPJ}$ and characterize their possible antitumor activity, especially for HCC. To this end, we used structure-based in silico virtual screening (VS) followed by surface plasmon resonance and a PPIase inhibition assay to identify CyPJ inhibitors. Based on those results, we optimized antitumor activity of CyPJ inhibitors using quinoxaline nucleus-associated rational design. The evidence derived from the current study provides novel potential $\mathrm{CyPJ}$ inhibitors and highlights the potent inhibitory effects of these compounds on tumor cell growth.

\section{MATERIALS AND METHODS}

\section{General Information}

Expression and purification of recombinant human CyPJ protein was carried out as described previously (Chen et al., 2015). CyPJ protein concentration was determined using the Bradford method. The substrates $N$-succinyl-Ala-Ala-Pro-Phep-nitroanilide (Suc-AAPF-pNA), CsA, 2,2,2-trifluoroethanol, and $\alpha$-chymotrypsin were purchased from Sigma (St. Louis, MO, United States). The libraries of small molecular compounds used for VS were obtained from SPECS (200,000 compounds $)^{1}$ and China Natural Products Database (CNPD, 50,000 compounds) ${ }^{2}$. The small organic compounds selected after VS were purchased from SPECS (Zoetermeer, Netherlands). Chemicals and solvents were either purchased from Sigma or purified by standard techniques. Analytical thin-layer chromatography (TLC) was performed on a Merck pre-coated TLC plate (silica gel 60 $\mathrm{F}_{254}$ ). Melting points were recorded on an $\mathrm{X}_{4}$-Data microscopic melting point apparatus and were uncorrected. Electrospray ionization-mass spectrometry (ESI-MS) was performed on a Bruker Esquire 3000 plus spectrometer. ${ }^{1} \mathrm{H}$ NMR spectra were recorded on a Bruker Avance 400 spectrometer in $\mathrm{CDCl}_{3}$ using tetramethylsilane (TMS) as the internal standard. Elemental analysis was performed on Carlo-Erba 1106 (Carlo Erba, Italy).

\section{Molecular Docking and Active Site Analysis}

Previously, we obtained two crystal structures of CyPJ: the CyPJ/CsA complex at $2.4 \AA$ (PDB: 2OJU) and the CyPJ crystal alone at $2.0 \AA$ (PDB: 2OK3) (Chen et al., 2015). In order to better mimic the affinity between $\mathrm{CyPJ}$ and the relevant compounds, and accurately calculate their docking scores, we selected the crystal structure of the CyPJ/CsA complex (2OJU) for molecular docking analysis. Arg44, Gln52, Asn92, His110, and Tyr115 were conserved active site residues located in the

\footnotetext{
${ }^{1}$ http://www.specs.net

${ }^{2}$ http://www.neotrident.com
} 
shallow pocket of the protein structure, where Arg44 displayed a flexible conformation. Our compounds of interest were then each flexibly docked on the protein structure. In our docking calculation, potential energy maps of the receptor were calculated using default parameters.

\section{In Silico Virtual Screening}

Virtual screening in silico was carried out as described elsewhere with minor modifications ( $\mathrm{Li}$ et al., 2006b). The structure of CyPJ/CsA complex (PDB: 2OJU) was used as a target. The DOCK 4.0 program suite was employed for primary screening of the small molecule databases SPECS and CNPD via 64CPU-SGI ORIGIN3800 (State Key Laboratory of computeraided drug design, Shanghai Institute of Materia Medica, Chinese Academy of Sciences, Shanghai, China) and 392CPU-Shenwei I supercomputer (Shanghai Supercomputing Center) (Kuntz, 1992; Butler et al., 2016; Choi et al., 2017). Based on the X-ray structure of the CyPJ/CsA complex (Chen et al., 2015), Arg44, Gln52, Asn92, His110, and Tyr115 residues surrounding CsA were selected to generate a cavity. Our small molecules were then docked into this cavity, and ligand-binding quality was evaluated using a force-field scoring function. During the docking calculation, Kollman united-atom charges were assigned to the CyPJ protein, and Gasteiger-Marsili partial charges were assigned to the small molecules in the databases. Conformational flexibility of the compounds from the databases was considered during the docking search. Three thousand topscoring compounds obtained by DOCK search were rescored by using the Consensus Score (CScore) method encoded in Sybyl6.8 (Sybyl molecular modeling package, version 6.8) (Houston and Walkinshaw, 2013; Campagna-Slater et al., 2014; Weill et al., 2014). Molecules with a CScore of $\geq 4$ were visually analyzed. Finally, 74 compounds with the lowest energy and most favorable ligand orientation were selected.

\section{Surface Plasmon Resonance (SPR) Assay}

The binding affinity of the selected compounds to CyPJ was measured by SPR with a Biacore 3000 instrument (Biacore AB Corporation, Uppsala, Sweden) as previously described (Guo et al., 2005; Chen et al., 2007). Briefly, recombinant human CyPJ protein was immobilized on an activated sensor chip via amine coupling. CyPJ protein $(10 \mu \mathrm{M})$ was coupled to a CM5 sensor chip surface (a carboxymethylated dextran surface; CM5 chip from Biacore, Inc., Piscataway, NJ, United States) in a buffer containing $10 \mathrm{mM}$ sodium acetate $(\mathrm{pH} 4.0)$ using standard amine coupling chemistry according to the manufacturer's instructions. Flow cell 1 (FC-1) was used as the control surface and flow cell 2 (FC-2) contained 9000 resonance units (RU) of CyPJ (1 RU corresponds to $1 \mathrm{pg}$ of protein per $\mathrm{mm}^{2}$ ). The buffer stream was passed through FC-1 and FC-2 of the bi-channel at a flow rate of $40 \mu \mathrm{l} / \mathrm{min}$. Compounds to be tested were purchased from SPECS (Netherlands). All chemicals were of the highest purity available, as certified by the vendor. All compounds were stocked in $100 \%$ dimethylsulfoxide at $10 \mathrm{mM}$ and diluted at graded concentrations $(0.3-10 \mu \mathrm{M})$ with HBS-EP buffer $(0.01 \mathrm{M}$
HEPES buffer, pH 7.4, containing $0.15 \mathrm{M} \mathrm{NaCl}, 3.4$ mM EDTA, and $0.005 \%$ surfactant P20) containing $1 \%$ dimethylsulfoxide. The temperature of the instrument was set to $20^{\circ} \mathrm{C}$, and the flow rate was set to $40 \mu \mathrm{l} / \mathrm{min}$. Each compound $(50 \mu \mathrm{l})$ was injected sequentially. $\mathrm{NaOH}(50 \mathrm{mM})$ was used to regenerate the surface of the CM5 chip. The 1:1 Langmuir binding model was used to determine the equilibrium dissociation constant $\left(K_{\mathrm{D}}\right)$. For fast interactions, the steady-state model was used to determine $K_{\mathrm{D}}$ values. All experiments were carried out in triplicate.

\section{PPlase Assay}

The PPIase assay was carried out with minor modifications as described previously (Kofron et al., 1991). We used an $\alpha$-chymotrypsin-coupled PPIase assay, where the substrate succinyl-Ala-Ala-Pro-Phe-p-nitroanilide (Suc-AAPF-pNA) is first converted to the trans conformation by PPIase, and can then be digested by $\alpha$-chymotrypsin to release chromogenic $p$-nitroanilide; the latter compound is then monitored with a spectrometer. Briefly, the assay was performed under $8^{\circ} \mathrm{C}$ in a $100 \mu \mathrm{l}$ system. The Suc-AAPF-pNA was dissolved in the tetrafluoroethylene containing $480 \mathrm{mM}$ of $\mathrm{LiCl}$ (working concentration: $3.0 \mathrm{mM}$ ). The $\alpha$-chymotrypsin was dissolved in $1 \mathrm{mM} \mathrm{HCl}$ (working concentration: $1.7 \mathrm{mM}$ ). When assayed, each test compound was diluted in $94 \mu \mathrm{l}$ of assay buffer $(50 \mathrm{mM}$ HEPES, $100 \mathrm{mM} \mathrm{NaCl} ; \mathrm{pH} 8.0$ at $0^{\circ} \mathrm{C}$ ), and then mixed with $2 \mu \mathrm{l}$ of CyPJ solution $(5 \mu \mathrm{M})$. After equilibrating on ice for $3 \mathrm{~h}$, $2 \mu \mathrm{l}$ of $\alpha$-chymotrypsin solution and $2 \mu \mathrm{l}$ of Suc-AAPF-pNA were added to the assay mixture, and the absorbance at the wavelength $390 \mathrm{~nm}$ was recorded for $20 \mathrm{~s}$ on a Jasco V-550 Spectrophotometer (Jasco, Inc., Easton, MD, United States). Three independent experiments were performed for each test compound and the respective half maximal inhibitory concentration $\left(\mathrm{IC}_{50}\right)$ was calculated with OriginPro 7.5 software (OriginLab, Northampton, MA, United States).

\section{Synthesis of 2,3-Substituted Quinoxaline-6-Amine (Compound 3)}

Briefly, benzaldehyde or furaldehyde $(0.1 \mathrm{~mol})$ was catalyzed by thiamine (vitamin B1, $0.02 \mathrm{~mol}$ ) to generate 2-hydroxy1,2-diphenylethanone or 1,2-di(furan-2-yl)-2-hydroxyethanone, respectively. The resulting alcohol hydroxyls were oxidized by $\mathrm{Cu}\left(\mathrm{NO}_{3}\right)_{2}$ to generate diones $\mathbf{1 a}$ and $\mathbf{1 b}$ in $\mathrm{HOAc} / \mathrm{H}_{2} \mathrm{O}(1: 1, \mathrm{~V} / \mathrm{V})$ at $70^{\circ} \mathrm{C}$ in $6 \mathrm{~h}$. The selective acylation of pyrrole was catalyzed with oxalyl chloride in carbon disulfide to generate the dione 1c at $-70^{\circ} \mathrm{C}$. These 1,2 -ethanedione derivatives (1a, $\mathbf{1 b}$, and 1c, $0.3 \mathrm{~mol}$ each) were mixed with 4 -nitro-o-phenylenediamine $(0.5 \mathrm{~mol})$ in $\mathrm{AcOH}(100 \mathrm{ml})$, and stirred at $110^{\circ} \mathrm{C}$ under normal atmosphere for $8 \mathrm{~h}$. The mixture was then cooled to room temperature and poured into water $(300 \mathrm{ml})$ to obtain 6nitro-2, 3-disubstituted quinoxaline (compound 2). A reaction bottle was charged with compound $2(0.1 \mathrm{~mol})$ and $\mathrm{Na} 2 \mathrm{~S} x$ (0.15 equiv) in ethanol $(30 \mathrm{ml})$ under normal atmosphere. The mixture was vigorously stirred by refluxing for $12 \mathrm{~h}$, and cooled to room temperature and poured into water $(100 \mathrm{ml})$ after competition. The mixture was next extracted with $20 \mathrm{ml}$ of 
EtOAc three times. The combined organic layer was washed with brine $(20 \mathrm{ml})$, dried with $\mathrm{Na}_{2} \mathrm{SO}_{4}$, and the solvent was removed under reduced pressure. Finally, compound $\mathbf{3}$ was purified as an eluent by flash column chromatography using $\mathrm{PE} / \mathrm{EtOAc}$.

\section{Synthesis of 2,3-Substituted Quinoxaline-6-Amine Derivatives (ZX-J-19 Derivatives)}

The synthesis of 2,3-substituted quinoxaline-6-amine derivatives (i.e., ZX-J-19 derivatives) was performed as described elsewhere (Chen et al., 2011; Sridevi et al., 2011). Briefly, compound 3 (20 mmol) was treated with acyl chloride $(30 \mathrm{mmol})$ in the presence of pyridine at room temperature in acetone. After compound 3 disappeared on TLC, the reaction mixture was poured into the water $(50 \mathrm{ml})$, immediately cooled to room temperature, filtered, and purified to collect $\mathbf{Z X}$-J-19 derivatives by flash column chromatography.

\section{Cell Lines and MTT-Based Cell Proliferation Assay}

Human HCC cells SK-HEP1 and QGY; breast cancer cells HCC1954, BT474, and MDA-MB468; ovarian cancer cells SKOV3; and prostate cancer cells PC3 and LNCaP were purchased from the Cell Bank of Chinese Academy of Sciences (Shanghai, China), and cultured in T75 flasks with complete DMEM or RPMI 1640 medium (GIBCO, Invitrogen, Gaithersburg, CA, United States) supplemented with 10\% FBS (Hyclone, New Zealand), and 1\% penicillin/streptomycin (Solarbio, Beijing, China) at $37^{\circ} \mathrm{C}$ in a humidified atmosphere containing $5 \% \mathrm{CO}_{2}$.

Cell proliferation was determined using methylthiazolyl diphenyl-tetrazolium bromide assay (MTT; Sigma-Aldrich). The cells were seeded onto 96-well plates in a total volume of $150 \mu \mathrm{l}$ at a density of $3.5 \times 10^{4}$ cells/well. After incubation for $24 \mathrm{~h}$, cells were treated with the selected compounds or positive control drugs 5-fluorouracil (5-FU) and CsA at the indicated dosages $(0,1.0,5.0,10,50$, and $250 \mu \mathrm{M})$. To avoid the effect of the solvent, the concentration of DMSO was less than $0.1 \%(\mathrm{v} / \mathrm{v})$ in all experiments. After incubation at $37^{\circ} \mathrm{C}$ for $48 \mathrm{~h}, 10 \mu \mathrm{l}$ of MTT solution $(5 \mathrm{mg} / \mathrm{ml}$ ) was added and continued to incubate for $4 \mathrm{~h}$. Following removal of medium containing MTT, $200 \mu \mathrm{l}$ of DMSO was added to dissolve the formazan crystals formed by live cells. Solution absorbance was measured at $490 \mathrm{~nm}$ with an absorbance microplate reader (BioTek, Winooski, VT, United States). The assay was repeated six times. The $\mathrm{IC}_{50}$ value was determined from plot of $\%$ viability against the dose of compounds added.

\section{Statistical Analysis}

The compound activities and MTT-based cell assays were repeated at least three times and the significance was assessed with unpaired Student's $t$-test. The survival curve was created by the Kaplan-Meier method and analyzed by the log-rank test. A $P<0.05$ was considered statistically significant.

\section{RESULTS}

\section{Identification of Small Molecule Inhibitors of CyPJ by Virtual Screening and SPR Analysis}

To identify the inhibitory small molecules of CyPJ, first VS was conducted, in silico, using commercially available compound libraries. Crystal structures of $\mathrm{CyPJ}$ complexed with CsA were analyzed for potential inhibitory ligands (Huang et al., 2005; Chen et al., 2015). As illustrated in Figure 1, we screened SPECS and CNPD databases with a total of 250,000 small molecule compounds to determine their ability to dock at the catalytic site of CyPJ. We selected 74 compounds with the best docking scores. Among them, 63 compounds were commercially available and purchased from SPECS and their CyPJ binding was subsequently analyzed by SPR.

Based on SPR analysis, 19 out of 63 compounds, i.e., ZX-J-1 to ZX-J-19 (Figure 2), were found to be capable of binding with CyPJ (Table 1). The binding of the above 19 compounds behaved in a concentration-dependent manner with $K_{\mathrm{i}}$ values ranging

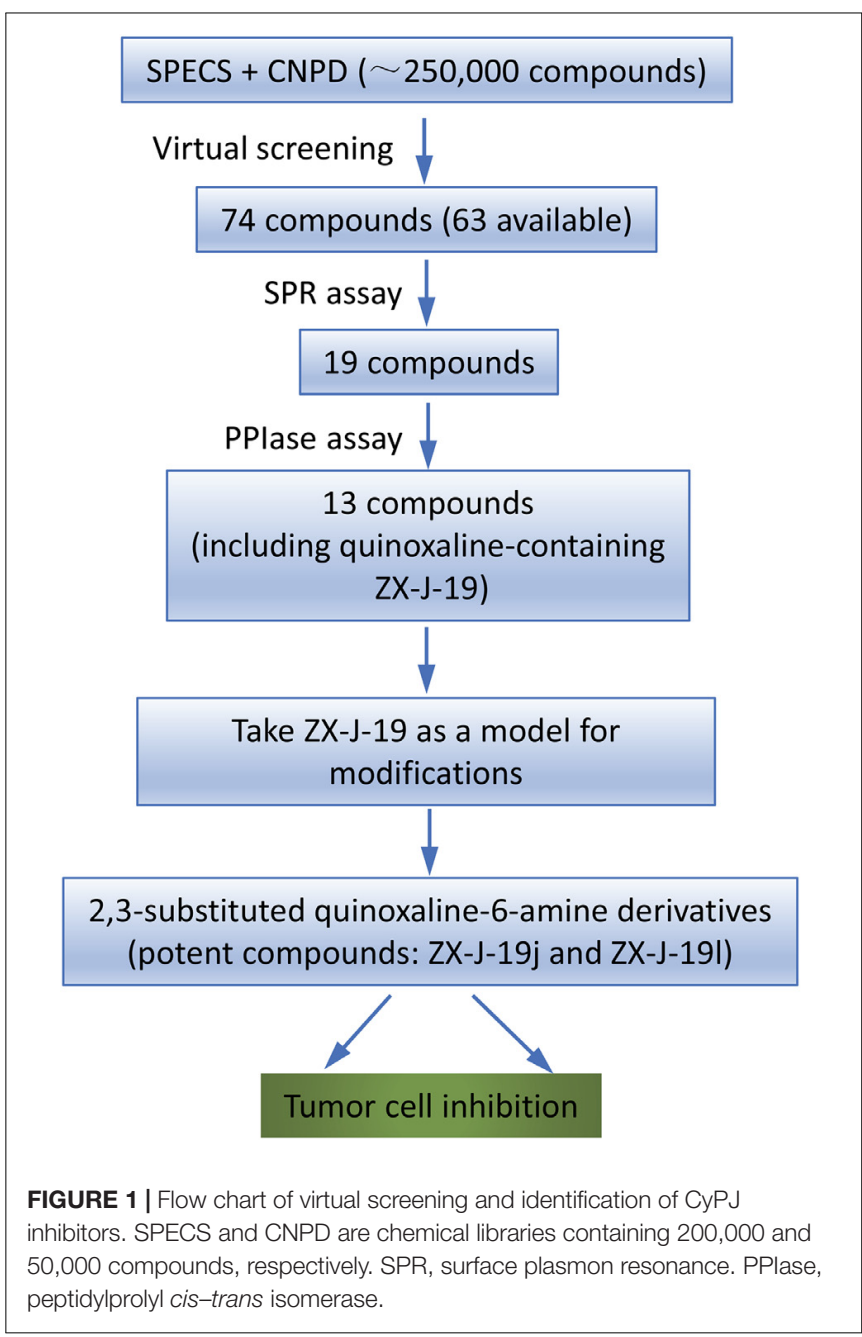




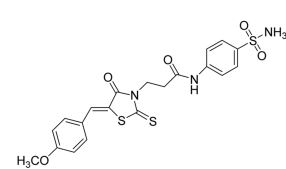

ZX-J-1

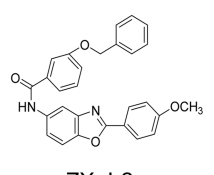

ZX-J-2
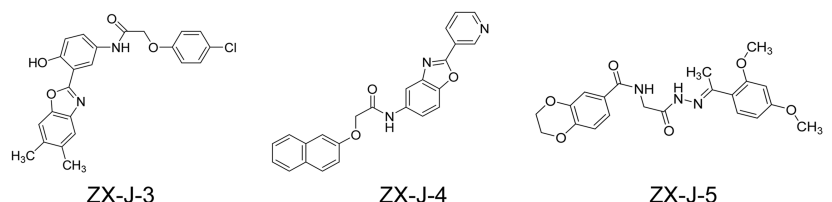

ZX-J-3

ZX-J-4

ZX-J-5
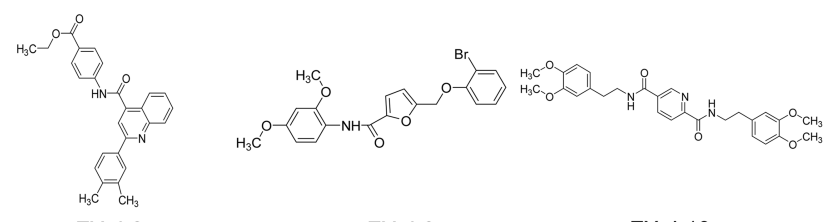

ZX-J-9

ZX-J-10

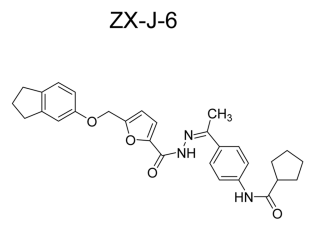

ZX-J-7

ZX-J-8
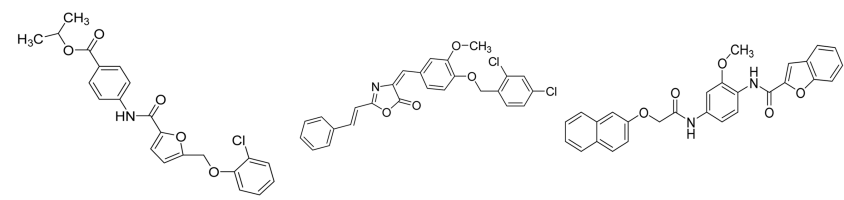

ZX-J-11

ZX-J-12

ZX-J-13

ZX-J-14

ZX-J-15

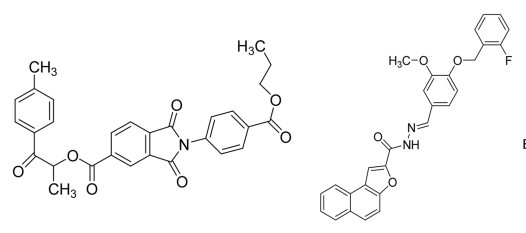

ZX-J-17

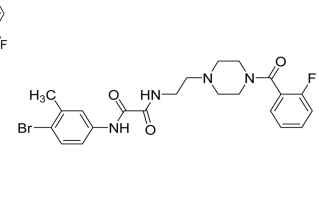

ZX-J-18
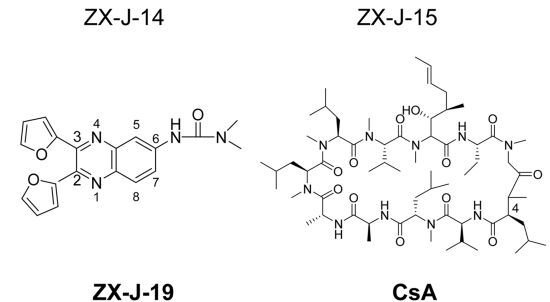

CsA

FIGURE 2 | Chemical structures of 19 potential CyPJ inhibitors identified with in silico screening and SPR analysis. Following in silico screening and SPR analysis, 19 ZX-J compounds numbered $\mathbf{1 - 1 9}$ were identified and selected for further investigation. The numbers in the compound $\mathbf{Z X - J - 1 9}$ represent the positions of the carbons in the quinoxaline ring. CsA, cyclosporine A, is a well-established cyclophilin protein inhibitor.

from $1 \times 10^{-4} \mathrm{M}$ to $9 \times 10^{-9} \mathrm{M}$ by VS, and with $K_{\mathrm{D}}$ values ranging from $1 \times 10^{-4} \mathrm{M}$ to $9 \times 10^{-8} \mathrm{M}$ by SPR analysis (Table 1). These results suggested that these 19 compounds strongly interact with CyPJ.

\section{Inhibition of the PPlase Activity by 13 Identified Compounds}

To investigate whether the selected compounds inhibit the PPIase activity of CyPJ, we performed a PPIase assay as described previously (Chen et al., 2015). The inhibitory effect of each compounds was determined with a standard spectrophotometric method in chymotrypsin-coupled assays with different concentrations of peptide substrate. The rate constants for the cis-trans conversion were evaluated by fitting the data to the integrated first-order rate equation through nonlinear least-square analysis. Since CyPJ belongs to the cyclophilin protein family, the inhibitory effect of these compounds appears to follow Michaelis-Menten kinetics (Chen et al., 2015). There were 13 compounds including ZX-J-1, ZX-J-2, ZX-J-4, ZX-J-5, ZX-J-6, ZX-J-7, ZX-J-8, ZX-J-9, ZX-J-11, ZX-J-12, ZX-J-13, ZX-J-16, and ZX-J-19 that had $\mathrm{IC}_{50}$ values ranging from 5 to $\sim 20 \mu \mathrm{M}$, slightly higher than that of CsA (Table 1). Meanwhile, no inhibition of PPIase activity of CyPJ was detectable in the remaining six compounds, $\mathbf{Z X}-\mathbf{J}-\mathbf{3}$, ZX-J-10, ZX-J-14, ZX-J-15, ZX-J-17, and ZX-J-18 (Table 1). We concluded that 13 out of 19 compounds may be regarded as the inhibitors of CyPJ.

\section{HCC Tumor Cell Growth Inhibition by Quinoxaline-Containing Compound ZX-J-19}

Among the 13 inhibitory compounds identified previously, ZX-J-19 is a quinoxaline derivative composed of a quinoxaline nucleus with a furanyl group at positions 2 and 3 and a urea group at position 6 (Figure 2). Since the quinoxaline nucleus may potentially have antitumor activity (Supplementary Table S1), together with our previous observation that siRNAbased CyPJ targeting inhibited tumor growth of HCC (Chen et al., 2015), we wanted to know whether the quinoxalinecontaining ZX-J-19 can inhibit HCC tumor cell growth. First of all, we verified the alterations of $C y P J$ in HCC in a large cohort from The Cancer Genome Atlas (TCGA). We found a higher CyPJ gene copy number in about $15 \%$ of HCC samples $(n=370)$ (Supplementary Figure S1A). Also, the $C y P J$ alterations were positively correlated with poor disease-free survival in HCC patients $(P=0.0287)$ (Supplementary Figure $\mathrm{S} 1 \mathrm{~B}$ ), suggesting that $\mathrm{CyPJ}$ is of importance in HCC.

Next, the inhibitory effects of $\mathbf{Z X}$-J-19 were determined using MTT-based in vitro cell proliferation assay along with CsA 
TABLE 1 | Affinity of inhibitors to CyPJ, and their respective $I C_{50}$ for CyPJ PPlase inhibition.

\begin{tabular}{|c|c|c|c|c|}
\hline Compound & Molecular weight (Da) & $\begin{array}{l}\text { CyPJ binding by virtual } \\
\text { screening } K_{\mathrm{i}}(\mathrm{M})^{\mathrm{a}}\end{array}$ & $\begin{array}{l}\text { CyPJ binding by SPR } \\
\qquad K_{\mathrm{D}}(\mathrm{M})^{\mathrm{b}}\end{array}$ & $\begin{array}{l}\text { Compound } I_{50} \\
\text { for PPlase }(\mu M)^{c}\end{array}$ \\
\hline ZX-J-1 & 477.584 & $1.30 \times 10^{-6}$ & $6.50 \times 10^{-6}$ & $15.949 \pm 0.062^{* *}$ \\
\hline ZX-J-2 & 450.492 & $8.38 \times 10^{-7}$ & $1.35 \times 10^{-6}$ & $8.944 \pm 0.027^{* *}$ \\
\hline ZX-J-3 & 422.866 & $8.03 \times 10^{-8}$ & $1.06 \times 10^{-6}$ & $-d$ \\
\hline ZX-J-4 & 395.416 & $3.54 \times 10^{-9}$ & $1.05 \times 10^{-7}$ & $11.376 \pm 0.768^{* *}$ \\
\hline ZX-J-5 & 413.428 & $7.45 \times 10^{-7}$ & $1.37 \times 10^{-6}$ & $21.324 \pm 0.911^{* * *}$ \\
\hline ZX-J-6 & 465.345 & $1.26 \times 10^{-9}$ & $2.85 \times 10^{-5}$ & $17.883 \pm 0.585^{* *}$ \\
\hline ZX-J-7 & 444.872 & $3.28 \times 10^{-8}$ & $1.26 \times 10^{-7}$ & $5.815 \pm 0.613^{* *}$ \\
\hline ZX-J-8 & 424.498 & $9.41 \times 10^{-9}$ & $1.45 \times 10^{-5}$ & $10.346 \pm 0.442^{*}$ \\
\hline ZX-J-9 & 432.268 & $4.76 \times 10^{-8}$ & $1.29 \times 10^{-6}$ & $8.542 \pm 0.965^{*}$ \\
\hline ZX-J-10 & 493.557 & $2.19 \times 10^{-6}$ & $8.00 \times 10^{-7}$ & $-d$ \\
\hline ZX-J-11 & 485.581 & $3.79 \times 10^{-7}$ & $9.88 \times 10^{-8}$ & $19.949 \pm 0.025^{* * *}$ \\
\hline ZX-J-12 & 454.523 & $1.62 \times 10^{-8}$ & $1.18 \times 10^{-4}$ & $12.952 \pm 0.054^{* * *}$ \\
\hline ZX-J-13 & 413.855 & $8.88 \times 10^{-8}$ & $5.16 \times 10^{-7}$ & $12.634 \pm 0.068^{* *}$ \\
\hline ZX-J-14 & 480.345 & $1.80 \times 10^{-7}$ & $1.12 \times 10^{-6}$ & $-d$ \\
\hline ZX-J-15 & 466.491 & $9.66 \times 10^{-8}$ & $6.61 \times 10^{-7}$ & $-d$ \\
\hline ZX-J-16 & 499.517 & $1.01 \times 10^{-4}$ & $3.41 \times 10^{-6}$ & $18.456 \pm 0.727^{* *}$ \\
\hline$Z X-J-17$ & 468.482 & $2.29 \times 10^{-7}$ & $4.35 \times 10^{-8}$ & $-d$ \\
\hline ZX-J-18 & 491.359 & $5.48 \times 10^{-9}$ & $2.42 \times 10^{-7}$ & $-d$ \\
\hline ZX-J-19 & 348.000 & $2.01 \times 10^{-6}$ & $1.67 \times 10^{-5}$ & $15.949 \pm 0.012^{* *}$ \\
\hline CsA & 1202.600 & n.d. & n.d. & $4.929 \pm 0.254$ \\
\hline
\end{tabular}

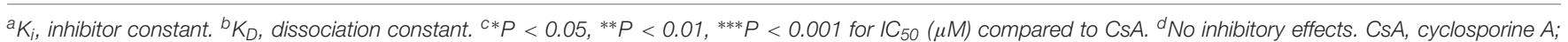
n.d., not determined.

(positive control for inhibiting CyPJ) and 5-FU (positive control for antitumor activity) on HCC cells SK-HEP1 and QGY. As shown in Table 2, the $\mathrm{IC}_{50}$ of ZX-J-19 on SK-HEP1 and QGY HCC cells was 40.440 and $52.438 \mu \mathrm{M}$, respectively. Although these $\mathrm{IC}_{50}$ values were slightly higher than those of CsA (10.243 and $7.902 \mu \mathrm{M}$, respectively), as the positive control inhibitor of CyPJ, they were strikingly lower than those of 5-FU (177.238 and $238.528 \mu \mathrm{M}$, respectively), a conventional clinical drug (Table 2), suggesting that ZX-J-19 potently inhibits HCC tumor cell growth in vitro.

\section{Modifications of 2,3-Substituted-Quinoxaline-6-Amine Derivatives with Synthetic Chemistry}

To identify more potent quinoxaline-containing compounds and further optimize them for tumor cell inhibition, we modified the quinoxaline nucleus at positions 2, 3, and 6 based on the structure of $\mathbf{Z X}-\mathbf{J}-\mathbf{1 9}$ using the rational-design strategy.

TABLE 2 | IC $\mathrm{C}_{50}$ of selected compounds on HCC cells.

\begin{tabular}{llrc}
\hline \multirow{2}{*}{ Entry } & \multirow{2}{*}{ Compounds } & \multicolumn{2}{c}{ IC $_{\mathbf{5 0}}(\boldsymbol{\mu} \mathbf{M})$} \\
\cline { 3 - 4 } & & \multicolumn{1}{c}{ SK-HEP1 } & QGY \\
\hline 1 & ZX-J-19 & $40.440 \pm 0.033$ & $52.438 \pm 0.019^{\mathrm{a}, \mathrm{b}}$ \\
2 & $5-\mathrm{FU}$ & $177.238 \pm 0.065$ & $238.528 \pm 0.035$ \\
3 & CSA & $10.243 \pm 0.082$ & $7.902 \pm 0.022$ \\
\hline
\end{tabular}

${ }^{a} P<0.05$ compared to CsA. ${ }^{b} P<0.05$ compared to $5-F U$.
First, we synthesized the ethanediones (compound 1, e.g., 1a, $\mathbf{1 b}$, and $\mathbf{1 c}$ ) as presented in Figure $\mathbf{3 A}$, which provided the necessary diversity of the $R_{1}$ residues at positions 2 and 3 . Benzaldehyde and furaldehyde reactions were catalyzed by thiamine to generate 2-hydroxy-1,2-diphenylethanone and 1,2-di (furan-2-yl)-2-hydroxyethanone, respectively (yields: 88 and $84 \%$, respectively). The resulting hydroxyethanone derivatives were then oxidized by $\mathrm{Cu}\left(\mathrm{NO}_{3}\right)_{2}$ to generate the diones $\mathbf{1 a}$ and 1b in $\mathrm{HOAc} / \mathrm{H}_{2} \mathrm{O}(1: 1, \mathrm{~V} / \mathrm{V})$ at $70^{\circ} \mathrm{C}$ in $6 \mathrm{~h}$ (yields: 91 and $87 \%$, respectively). For the synthesis of $\mathbf{1 c}$, selective acylation of pyrrole was catalyzed with oxalyl chloride in carbon disulfide at $-70^{\circ} \mathrm{C}$ (yield: $81 \%$ ).

Next, 2,3-substituted-6-amine derivatives of quinoxaline were synthesized, and the synthesis route is illustrated in Figure 3B. The compounds $\mathbf{1 a}, \mathbf{1 b}$, or $\mathbf{1 c}$ were condensed with 4-nitro$o$-phenylene diamine in HOAc under reflux for about $8 \mathrm{~h}$ to generate 6-nitro-2,3-diphenyl quinoxaline, 2,3-di(furan-2-yl)-6nitroquinoxaline, or 6-nitro-2,3-di(1H-pyrrol-2-yl) quinoxaline (compound 2), respectively. The resulting nitro-quinoxalines (2) (e.g., 6-nitro-2,3-diphenyl quinoxaline) was reduced by $\mathrm{Na}_{2} \mathrm{~S} x$ (1.5 equiv) under reflux in ethanol to furnish the 2,3-diphenylquinoxaline-6-amine (compound 3). Compound 3 (1 equiv) was then treated with acyl chloride (1.3 equiv) in acetone at room temperature to produce the 22 2,3-quinoxaline6-amine (ZX-J-19) derivatives, as shown in Table 3 (ZX-J19a-ZX-J-19v; yield: 63-81\%). All synthesized compounds were structurally characterized by ${ }^{1} \mathrm{H}$ NMR, elemental analysis, and mass spectral analysis (see the Supplementary Structure Data listed in the Supplementary Materials). These compounds were categorized into three groups according to the property of the 
<smiles>O=Cc1ccccc1</smiles>

Benzaldehyde<smiles>O=Cc1ccco1</smiles>

Furaldehyde (a)<smiles>O=C(c1ccccc1)C(O)c1ccccc1</smiles>

2-hydroxy-1,2-diphenylethanone (b)<smiles>O=C(C(=O)c1ccccc1)c1ccccc1</smiles>

$1 \mathrm{a}$ (a)<smiles>O=C(c1ccco1)C(O)c1ccco1</smiles>

(b)<smiles>O=C(C(=O)c1ccco1)c1ccco1</smiles>

$1 \mathrm{~b}$

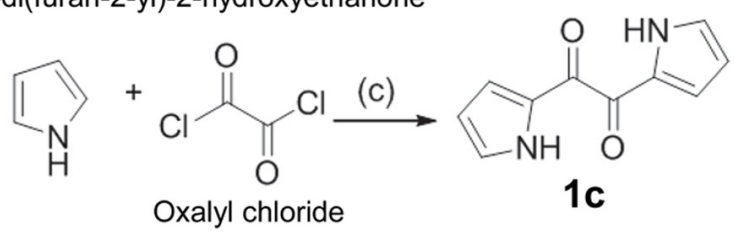

B<smiles>[R]C(=O)C([R])=[O+]</smiles><smiles>[R]c1nc2ccc(N)cc2nc1[R]</smiles>

3<smiles>[R]C(=O)Nc1ccc2nc([R])c([R])nc2c1</smiles>

ZX-J-19 derivatives

FIGURE 3 | The synthesis route of 2,3-substituted-quinoxaline-6-amine derivatives. (A) The synthetic production of ethanediones (including intermediate chemicals 1a, 1b, 1c). Reagents and conditions: (a) Vitamin B1 (VB1), EtOH/ $\mathrm{H}_{2} \mathrm{O}$ 2:1, $\mathrm{NaOH}$, room temperature, $12 \mathrm{~h} ;(\mathrm{b}) \mathrm{Cu}(\mathrm{NO})_{2}, \mathrm{HOAc}_{2} \mathrm{H}_{2} \mathrm{O} 1: 1,70^{\circ} \mathrm{C}, 6 \mathrm{~h} ;(\mathrm{c}) \mathrm{carbon}$ disulfide, $-70^{\circ} \mathrm{C}$, pyridine, $4 \mathrm{~h}$. (B) The synthetic process of 2,3-substituted-quinoxaline-6-amine derivatives (i.e., ZX-J-19 derivatives). Compound $\mathbf{1}$ was referred to as 1a, 1b, or 1c obtained from (A). Reagents and conditions: (a) HOAc, reflux, 8 h; (b) $\mathrm{Na}_{2} \mathrm{~S} x, x=2-6$, EtOH, reflux, 12 h; (c) RCOCl, pyridine, acetone, reflux, 4 h. The numbers in the $\mathbf{Z X}-\mathbf{J}-\mathbf{1 9}$ derivatives represent the position of the carbons in the quinoxaline ring. $\mathrm{R}_{1}$ refers to the side residuals at the positions 2 and 3 of the quinoxalines. $R_{2}$ refers to the side residual linked to the amide group at the position 6 of the quinoxalines.

residues at the $\mathrm{R}_{1}$ position: the phenyl type (ZX-J-19a-ZX-J191), the furanyl type (ZX-J-19m-ZX-J-19s), and the pyrrole type (ZX-J-19t-ZX-J-19v), and the compounds with the furanyl type were more closely related to compound ZX-J-19.

\section{Remarkable Inhibition of Tumor Cell Growth by ZX-J-19 Derivatives}

To determine the effects of the 22 ZX-J-19 derivatives (a-v) on tumor cell growth, we first examined their activity on HCC cells as detected by the MTT-based in vitro cell proliferation assay. As shown in Table 4, four compounds ZX-J-19e, ZX-J-19g, ZX-J-19j, and ZX-J-19l, all belonging to the phenyl type of 2,3-substituted quinoxaline-6-amine derivatives, exhibited remarkable inhibitory effects on HCC cell growth. Their potency was comparable to the potency of the positive CyPJ inhibitor control, CsA, shown in Table 2. Among these four compounds, ZX-J-19j and ZX-J-19l exhibited the most potency. This result is evident by their lower $\mathrm{IC}_{50}$ on SK-HEP1 cells (6.725 and
$3.512 \mu \mathrm{M}$, respectively) (Table 4 ), suggesting that $\mathbf{Z X}-\mathbf{J}-\mathbf{1 9 j}$ and ZX-J-191 may be better than ZX-J-19 itself and other ZX-J-19 derivatives in HCC cell growth inhibition.

Besides HCC, the CyPJ gene is also aberrantly dysregulated in several other malignancies such as breast, prostate, and ovarian cancers (Supplementary Figure S2) (Qi et al., 2005; Couch et al., 2016; Gong et al., 2017). With this fact in mind, we further determined if ZX-J-19j and ZX-J-19l could inhibit growth of tumor cells originating from other cancers, including breast cancer cells HCC1954, BT474, and MDA-MB468; ovarian cancer cells SKOV3; and prostate cancer cells PC3 and LNCaP. As shown in Table 5, both ZX-J-19j and ZX-J-191 showed significant growth inhibition in these tumor cells, suggesting ZX-J-19j and ZX-J-19l might inhibit tumor cell growth in a wide spectrum of malignancies.

It should be pointed out that although $\mathbf{Z X}-\mathbf{J}-\mathbf{1 9} \mathbf{j}$ and ZX-J-191 demonstrated convincing inhibitory effects on tumor cell growth by using in vitro cell models, these two 
TABLE 3 | Isolated yields of 2,3-substituted quinoxaline-6-amine derivatives.

\begin{tabular}{|c|c|c|c|}
\hline ZX-J-19 derivatives ${ }^{a}$ & $\mathbf{R}_{1}{ }^{b}$ & $\mathbf{R}_{\mathbf{2}}{ }^{c}$ & Isolated yield (\%) \\
\hline a & Phenyl & $-\mathrm{CH}_{2}-\mathrm{Ph}$ & 63 \\
\hline$b$ & Phenyl & $-\mathrm{C}(\mathrm{Me})_{3}$ & 71 \\
\hline C & Phenyl & -(p-nitrophenyl) & 65 \\
\hline d & Phenyl & $-\mathrm{CH}_{2} \mathrm{Cl}$ & 79 \\
\hline e & Phenyl & -(m-chlorophenyl) & 64 \\
\hline f & Phenyl & -(o-chlorophenyl) & 67 \\
\hline g & Phenyl & -(p-chlorophenyl) & 73 \\
\hline $\mathrm{h}$ & Phenyl & $-\mathrm{Ph}$ & 81 \\
\hline i & Phenyl & $-\mathrm{C}_{2} \mathrm{H}_{5}$ & 78 \\
\hline j & Phenyl & $-\mathrm{C}_{7} \mathrm{H}_{15}$ & 63 \\
\hline k & Phenyl & $-\mathrm{CH}_{3}$ & 74 \\
\hline । & Phenyl & $-\mathrm{C}_{3} \mathrm{H}_{7}$ & 76 \\
\hline $\mathrm{m}$ & 2-Furanyl & $-\mathrm{C}(\mathrm{Me})_{3}$ & 73 \\
\hline$n$ & 2-Furanyl & $-\mathrm{C}_{3} \mathrm{H}_{7}$ & 70 \\
\hline $\mathrm{O}$ & 2-Furanyl & $-\mathrm{C}_{7} \mathrm{H}_{15}$ & 67 \\
\hline$p$ & 2-Furanyl & -(o-chlorophenyl) & 65 \\
\hline$q$ & 2-Furanyl & -(p-nitrophenyl) & 63 \\
\hline r & 2-Furanyl & $-\mathrm{Ph}$ & 80 \\
\hline s & 2-Furanyl & $-\mathrm{CH}_{2}-\mathrm{Ph}$ & 77 \\
\hline t & 2-Pyrrole & -(p-chlorophenyl) & 65 \\
\hline $\mathrm{u}$ & 2-Pyrrole & $-\mathrm{CH}_{3}$ & 69 \\
\hline v & 2-Pyrrole & $-\mathrm{CH}_{2} \mathrm{Cl}$ & 71 \\
\hline
\end{tabular}

${ }^{a} A$ total of 22 compounds (ZX-J-19a-ZX-J-19v) isolated. ${ }^{b}$ Two $R_{1}$ groups residing at positions 2 and 3 of the quinoxaline ring, respectively. ${ }^{C} R_{2}$ group was linked to the amide at position 6 of the quinoxaline ring. Ph, phenyl; Me, methyl.

compounds still retain similar lipophilicity to ZX-J-19. This characteristic may contribute to poor antitumor activity of ZX-J-19 in an in vivo xenograft mouse tumor model due to its poor pharmacokinetics (unpublished data). For example, this compound was absorbed and accumulated in blood serum within $10 \mathrm{~min}$ after intraperitoneal administration, peaked within 30-60 min, and then declined and diminished to control levels after $8 \mathrm{~h}$. These results suggest that these 2,3substituted quinoxaline- 6 amine compounds need to be further optimized for in vivo application, a finding which is under investigation.

Taken together, our data suggest that these quinoxalinecontaining derivatives (e.g., ZX-J-19j and ZX-J-191) are lead compounds for novel CyPJ-targeting antitumor drugs.

\section{DISCUSSION}

Based on the three-dimensional structure of CyPJ reported in our previous research (Hu et al., 2005; Huang et al., 2005; Chen et al., 2015), we virtually screened the chemical libraries of SPECS and CNPD in silico, and were able to identify 13 inhibitors of CyPJ (Table 1). Because of the well-characterized potential antitumor activity of quinoxaline derivatives and their relative ease of synthesis, we focused on the quinoxaline-containing compound ZX-J-19 and quinoxalinebased modifications of that template. A total of 22 2,3substituted-quinoxaline-6-amine derivatives were successfully
TABLE 4 | IC 50 of ZX-J-19 derivatives on HCC cells.

\begin{tabular}{|c|c|c|c|}
\hline \multirow[t]{2}{*}{ Entry } & \multirow[t]{2}{*}{ ZX-J-19 derivatives } & \multicolumn{2}{|c|}{$I C_{50}(\mu M)^{a}$} \\
\hline & & SK-HEP1 & QGY \\
\hline 1 & $a$ & $387.602 \pm 0.044$ & $208.271 \pm 0.021$ \\
\hline 2 & $\mathrm{~b}$ & $21.595 \pm 0.031$ & $25.976 \pm 0.009$ \\
\hline 3 & C & $719.724 \pm 0.036$ & $37.644 \pm 0.079$ \\
\hline 4 & $d$ & $11.350 \pm 0.013$ & $17.943 \pm 0.033$ \\
\hline 5 & e & $7.495 \pm 0.057$ & $12.314 \pm 0.0146$ \\
\hline 6 & $f$ & $321.356 \pm 0.087$ & $541.697 \pm 0.0297$ \\
\hline 7 & $g$ & $9.890 \pm 0.047$ & $11.263 \pm 0.088$ \\
\hline 8 & $\mathrm{~h}$ & $40.332 \pm 0.024$ & $27.505 \pm 0.035$ \\
\hline 9 & i & $40.767 \pm 0.068$ & $588.584 \pm 0.087$ \\
\hline 10 & j & $6.725 \pm 0.023$ & $12.524 \pm 0.097$ \\
\hline 11 & $\mathrm{k}$ & $7.534 \pm 0.021$ & $198.752 \pm 0.005$ \\
\hline 12 & I & $3.512 \pm 0.022$ & $21.353 \pm 0.037$ \\
\hline 13 & $\mathrm{~m}$ & $76.744 \pm 0.017$ & $82.343 \pm 0.007$ \\
\hline 14 & $\mathrm{n}$ & $17.650 \pm 0.082$ & $18.022 \pm 0.015$ \\
\hline 15 & 0 & $17.334 \pm 0.041$ & $98.437 \pm 0.017$ \\
\hline 16 & $\mathrm{p}$ & $45.724 \pm 0.052$ & $548.538 \pm 0.042$ \\
\hline 17 & q & $43.648 \pm 0.021$ & $44.258 \pm 0.023$ \\
\hline 18 & r & $18.830 \pm 0.044$ & $17.315 \pm 0.015$ \\
\hline 19 & $s$ & $332.354 \pm 0.064$ & $416.804 \pm 0.027$ \\
\hline 20 & $\mathrm{t}$ & $46.348 \pm 0.033$ & $42.458 \pm 0.008$ \\
\hline 21 & $\mathrm{u}$ & $14.248 \pm 0.039$ & $32.669 \pm 0.019$ \\
\hline 22 & v & $140.343 \pm 0.028$ & $127.490 \pm 0.045$ \\
\hline
\end{tabular}

${ }^{a} / C_{50}$ values were determined using a MTT-based cell proliferation assay.

synthesized using a structure-activity relationship (SAR) strategy (Table 3). Moreover, we were able to provide convincing evidence that the growth in several tumor cell lines was inhibited by ZX-J-19j and ZX-J-19l (Tables 4, 5). These representatives of strong antitumor activity may act as lead compounds to facilitate the development of promising drugs targeting CyPJ-associated cancers.

In this study, we confirmed aberrations of the CyPJ gene previously reported in HCC (Chen et al., 2015) and demonstrated that its expression was associated with HCC progression (Supplementary Figure S1). Although CyPJ was relatively uninvestigated previously, a few reports suggested that it might play an important role in tumorigenesis and/or progression in HCC (Chen et al., 2015) and other cancers (Qi et al., 2005;

TABLE 5 | Growth inhibition of multiple cancer cell lines with compounds ZX-J-19j and ZX-J-19I.

\begin{tabular}{llll}
\hline \multirow{2}{*}{ Cell lines } & \multicolumn{2}{c}{$\%$ Growth inhibition at $\mathbf{2 0} \boldsymbol{\mu} \mathbf{M}$} \\
\cline { 3 - 4 } & & $\mathbf{Z X - J - 1 9 j}$ & $\mathbf{Z X - J - 1 9 l}$ \\
\hline HCC1954 & Breast cancer & 64.177 & 91.258 \\
BT474 & & 84.007 & 90.963 \\
MDA-MB-468 & & 84.653 & 90.849 \\
SKOV3 & Ovarian cancer & 48.067 & 90.919 \\
PC3 & Prostate cancer & 84.653 & 91.039 \\
LNCaP & & 48.067 & 91.038
\end{tabular}


Couch et al., 2016; Gong et al., 2017). For example, we have previously shown the importance of $C y P J$ expression in HCC; $\mathrm{CyPJ}$ inactivation by CsA- or siRNA-based $C y P J$ knockdown diminished HCC tumor cell growth in vitro and in vivo, demonstrating CyPJ was capable of initiating tumorigenesis of HCC (Chen et al., 2015). Our observations in this study are consistent with these previous findings (Supplementary Figure S1). Previous research provided support for the further investigation of CpPJ inhibitors ZX-J-19j and ZX-J-191 as potent inhibitors of tumor cell growth.

Cyclosporine $\mathrm{A}$ is a well-established potent inhibitor of cyclophilins that can also block the PPIase catalytic sites of CyPJ (Chen et al., 2015). CsA has profound implications in immunosuppression for organ transplantation (Colombo and Ammirati, 2011; Ziaei et al., 2016), however, as an antitumor agent, CsA may not be suitable due to potent side effects related to secondary skin cancers and other malignancies (Behnam et al., 2005; Gallagher et al., 2010; Norman et al., 2010; Han et al., 2012; Muellenhoff and Koo, 2012). It is therefore necessary to identify novel small molecule antitumor compounds that target cyclophilins including CyPJ. In this report, we identified at least 13 compounds, including the quinoxalinecontaining compound ZX-J-19, that exhibited notable inhibitory activities of PPIase in CyPJ (Table 1). Intriguingly, based on the quinoxaline nucleus scaffold of $\mathbf{Z X}-\mathbf{J}-19$, we rationally designed and ultimately synthesized 22 ZX-J-19-like quinoxaline derivatives with 2,3-substituted-6-amine modifications. Two of the resulting compounds ZX-J-19j and ZX-J-191 had notably enhanced antitumor activities (Tables 4, 5).

Consistent with these results, the quinoxaline nucleus is known to exhibit antitumor activity, and quinoxaline-derived compounds have been reported to have potential in cancer treatment (Zarranz et al., 2004; Amin et al., 2006; Weng et al., 2008; Pereira et al., 2015). For example, several quinoxaline-1,4di- $N$-oxide derivatives exhibited considerable antitumor activity in MCF-7 (breast), NCI-H460 (lung), and SF-268 (CNS) cells; such antitumor activity was dependent on the substituents at the carbonyl group (Zarranz et al., 2004). With regard to ZXJ-19j and ZX-J-19l, the phenyl at position $\mathrm{R}_{1}$ and the heptyl (ZX-J-19j) or propyl (ZX-J-19l) at position $\mathrm{R}_{2}$ were considered as the residues that contribute to the amelioration of antitumor capability. Similarly, our data indicated that at least ZX-J19j and ZX-J-19l are optimal lead candidate compounds for further development of novel CyPJ inhibitors and the potential antitumor drugs for CyPJ-based targeting.

As discussed previously, the quinoxaline derivative structures were determined based on CyPJ protein structure. However, we cannot rule out the possibility that these derivatives may impair the PPIase activity of other human cyclophilins. There are at least 15 members of the cyclophilin protein family (Fruman et al., 1994; Galat and Metcalfe, 1995). Due to high degree of similarity between members, cyclophilins inhibitors may have overlapping inhibitory action against many cyclophilins. For instance, a potent, well-characterized cyclophilin inhibitor, CsA, can inhibit the PPIase activities of almost all cyclophilins including CyPJ (Handschumacher et al., 1984; Chen et al., 2015). Cyclophilin CyPA has similarly been reported to be involved in many diseases; CyPA PPIase-based inhibitor Debio025 (Alisporivr) has been shown to inhibit several other cyclophilins including CyPB and CyPD (Flisiak et al., 2008; Quarato et al., 2012; Lee, 2013). Specifically for quinoxalinederived compounds, it has been reported that they may inhibit at least CyPA and CyPD (Guo et al., 2005; Li et al., 2006a). In this respect, it may be not unexpected that our CyPJ inhibitors may also inhibit other members of cyclophilins at some extent, and this issue will be the focus of future work.

In summary, we carried out a computer-aided virtual inhibitor screening of chemical databases based on the crystal structure of CyPJ/CsA complex, and identified 13 potential inhibitors of CyPJ. The quinoxaline-containing ZX-J-19, and its derivatives ZX-J-19j and ZX-J-19l showed potent antitumor activity. To the best of our knowledge, this study is the first to identify the inhibitors of CyPJ; these compounds are potential leads for rational design of novel CyPJ-based antitumor drugs.

\section{AUTHOR CONTRIBUTIONS}

XZ and LY designed the project. XZ, CX, XW, HW, MX, and YL performed the experiments and data analysis. XZ, CX, and YL wrote the manuscript. All authors discussed the results and revised the manuscript.

\section{FUNDING}

This work was supported by the grants from the National Natural Science Foundation of China (Grant Nos. 81172959 to XZ and 81372589 to YL), the Shandong Science and Technology Development Planning of China (Grant Nos. BS2009SW059 and 2007GG20002017, both to XZ), and the Shandong Medical and Health Science and Technology Development Planning of China (Grant No. 2011HZ104 to XZ).

\section{ACKNOWLEDGMENTS}

We are grateful to all members in the laboratory of Prof. Long Yu who provided technical assistance with SPR assay and Dr. Jinzhi Tan at the State Key Laboratory of Drug Research at the Chinese Academy of Sciences (Shanghai, China) for assisting in virtual screening.

\section{SUPPLEMENTARY MATERIAL}

The Supplementary Material for this article can be found online at: https://www.frontiersin.org/articles/10.3389/fphar. 2018.00126/full\#supplementary-material 


\section{REFERENCES}

Amin, K. M., Ismail, M. M., Noaman, E., Soliman, D. H., and Ammar, Y. A. (2006). New quinoxaline 1,4-di-N-oxides. Part 1: Hypoxia-selective cytotoxins and anticancer agents derived from quinoxaline 1,4-di-N-oxides. Bioorg. Med. Chem. 14, 6917-6923. doi: 10.1016/j.bmc.2006.06.038

Behnam, S. M., Behnam, S. E., and Koo, J. Y. (2005). Review of cyclosporine immunosuppressive safety data in dermatology patients after two decades of use. J. Drugs Dermatol. 4, 189-194.

Butler, K. V., Ma, A., Yu, W., Li, F., Tempel, W., Babault, N., et al. (2016). Structure-based design of a covalent inhibitor of the set domain-containing protein 8 (SETD8) lysine methyltransferase. J. Med. Chem. 59, 9881-9889. doi: 10.1021/acs.jmedchem.6b01244

Campagna-Slater, V., Therrien, E., Weill, N., and Moitessier, N. (2014). Methods for docking small molecules to macromolecules: a user's perspective. 2. applications. Curr. Pharm. Des. 20, 3360-3372. doi: 10.2174/13816128113199990606

Chen, J., Chen, S., Wang, J., Zhang, M., Gong, Z., Wei, Y., et al. (2015). Cyclophilin $\mathrm{J}$ is a novel peptidyl-prolyl isomerase and target for repressing the growth of hepatocellular carcinoma. PLoS One 10:e0127668. doi: 10.1371/journal.pone. 0127668

Chen, Q., Bryant, V. C., Lopez, H., Kelly, D. L., Luo, X., and Natarajan, A. (2011). 2,3-Substituted quinoxalin-6-amine analogs as antiproliferatives: a structure-activity relationship study. Bioorg. Med. Chem. Lett. 21, 1929-1932. doi: 10.1016/j.bmcl.2011.02.055

Chen, S., Zhao, X., Tan, J., Lu, H., Qi, Z., Huang, Q., et al. (2007). Structure-based identification of small molecule compounds targeting cell cyclophilin A with anti-HIV-1 activity. Eur. J. Pharmacol. 565, 54-59. doi: 10.1016/j.ejphar.2007. 03.023

Choi, J. Y., Fuerst, R., Knapinska, A. M., Taylor, A. B., Smith, L., Cao, X., et al. (2017). Structure-Based Design and Synthesis of Potent and Selective Matrix Metalloproteinase 13 Inhibitors. J. Med. Chem. 60, 5816-5825. doi: 10.1021/acs. jmedchem.7b00514

Colombo, D., and Ammirati, E. (2011). Cyclosporine in transplantation - a history of converging timelines. J. Biol. Regul. Homeost. Agents 25, 493-504.

Couch, F. J., Kuchenbaecker, K. B., Michailidou, K., Mendoza-Fandino, G. A., Nord, S., Lilyquist, J., et al. (2016). Identification of four novel susceptibility loci for oestrogen receptor negative breast cancer. Nat. Commun. 7:11375. doi: $10.1038 /$ ncomms11375

Dawar, F. U., Tu, J., Khattak, M. N., Mei, J., and Lin, L. (2017). Cyclophilin A: a key factor in virus replication and potential target for anti-viral therapy. Curr. Issues Mol. Biol. 21, 1-20.

Dunyak, B. M., and Gestwicki, J. E. (2016). Peptidyl-proline isomerases (PPIases): targets for natural products and natural product-inspired compounds. J. Med. Chem. 59, 9622-9644. doi: 10.1021/acs.jmedchem.6b00411

El Aissi, R., Liu, J., Besse, S., Canitrot, D., Chavignon, O., Chezal, J. M., et al. (2014). Synthesis and biological evaluation of new quinoxaline derivatives of ICF01012 as melanoma-targeting probes. ACS Med. Chem. Lett. 5, 468-473. doi: $10.1021 / \mathrm{ml} 400468 \mathrm{x}$

Flisiak, R., Horban, A., Gallay, P., Bobardt, M., Selvarajah, S., WiercinskaDrapalo, A., et al. (2008). The cyclophilin inhibitor Debio-025 shows potent anti-hepatitis $C$ effect in patients coinfected with hepatitis $C$ and human immunodeficiency virus. Hepatology 47, 817-826. doi: 10.1002/hep. 22131

Fruman, D. A., Burakoff, S. J., and Bierer, B. E. (1994). Immunophilins in protein folding and immunosuppression. FASEB J. 8, 391-400. doi: 10.1096/fasebj.8.6. 7513288

Galat, A., and Metcalfe, S. M. (1995). Peptidylproline cis/trans isomerases. Prog. Biophys. Mol. Biol. 63, 67-118. doi: 10.1016/0079-6107(94)00009-X

Gallagher, M. P., Kelly, P. J., Jardine, M., Perkovic, V., Cass, A., Craig, J. C., et al. (2010). Long-term cancer risk of immunosuppressive regimens after kidney transplantation. J. Am. Soc. Nephrol. 21, 852-858. doi: 10.1681/ASN. 2009101043

Gong, Z., Mu, Y., Chen, J., Chu, H., Lian, P., Wang, C., et al. (2017). Expression and significance of cyclophilin $\mathrm{J}$ in primary gastric adenocarcinoma. Anticancer Res. 37, 4475-4481.

Gothel, S. F., and Marahiel, M. A. (1999). Peptidyl-prolyl cis-trans isomerases, a superfamily of ubiquitous folding catalysts. Cell Mol. Life. Sci. 55, 423-436.
Gu, W., Wang, S., Jin, X., Zhang, Y., Hua, D., Miao, T., et al. (2017). Synthesis and evaluation of new quinoxaline derivatives of dehydroabietic acid as potential antitumor agents. Molecules 22:E1154. doi: 10.3390/molecules22071154

Guo, H. X., Wang, F., Yu, K. Q., Chen, J., Bai, D. L., Chen, K. X., et al. (2005). Novel cyclophilin D inhibitors derived from quinoxaline exhibit highly inhibitory activity against rat mitochondrial swelling and $\mathrm{Ca} 2+$ uptake/ release. Acta Pharmacol. Sin. 26, 1201-1211. doi: 10.1111/j.1745-7254.2005.00189.x

Han, W., Soltani, K., Ming, M., and He, Y. Y. (2012). Deregulation of XPC and CypA by cyclosporin A: an immunosuppression-independent mechanism of skin carcinogenesis. Cancer Prev. Res. 5, 1155-1162. doi: 10.1158/1940-6207. CAPR-12-0185-T

Handschumacher, R. E., Harding, M. W., Rice, J., Drugge, R. J., and Speicher, D. W. (1984). Cyclophilin: a specific cytosolic binding protein for cyclosporin A. Science 226, 544-547. doi: 10.1126/science.6238408

Harikishore, A., and Yoon, H. S. (2015). Immunophilins: structures, mechanisms and ligands. Curr. Mol. Pharmacol. 9, 37-47. doi: 10.2174/1874467208666150519113427

Hopkins, S., and Gallay, P. A. (2015). The role of immunophilins in viral infection. Biochim. Biophys. Acta 1850, 2103-2110. doi: 10.1016/j.bbagen.2014.11.011

Houston, D. R., and Walkinshaw, M. D. (2013). Consensus docking: improving the reliability of docking in a virtual screening context. J. Chem. Inf. Model. 53, 384-390. doi: 10.1021/ci300399w

Hu, H., Huang, C. Q., Liu, H. L., Han, Y., Yu, L., and Bi, R. C. (2005). Crystallization and preliminary X-ray crystallographic studies of human cyclophilin J. Acta Crystallogr. Sect. F Struct. Biol. Cryst. Commun. 61, 216-218. doi: 10.1107/ S1744309105000643

Huang, L. L., Zhao, X. M., Huang, C. Q., Yu, L., and Xia, Z. X. (2005). Structure of recombinant human cyclophilin J, a novel member of the cyclophilin family. Acta Crystallogr. D Biol. Crystallogr. 61, 316-321. doi: 10.1107/S0907444904033189

Hui, X., Desrivot, J., Bories, C., Loiseau, P. M., Franck, X., Hocquemiller, R., et al. (2006). Synthesis and antiprotozoal activity of some new synthetic substituted quinoxalines. Bioorg. Med. Chem. Lett. 16, 815-820. doi: 10.1016/j.bmcl.2005. 11.025

Kim, H., Kim, W. J., Jeon, S. T., Koh, E. M., Cha, H. S., Ahn, K. S., et al. (2005). Cyclophilin A may contribute to the inflammatory processes in rheumatoid arthritis through induction of matrix degrading enzymes and inflammatory cytokines from macrophages. Clin. Immunol. 116, 217-224. doi: 10.1016/j.clim. 2005.05.004

Kofron, J. L., Kuzmic, P., Kishore, V., Colon-Bonilla, E., and Rich, D. H. (1991). Determination of kinetic constants for peptidyl prolyl cis-trans isomerases by an improved spectrophotometric assay. Biochemistry 30, 6127-6134. doi: $10.1021 /$ bi00239a007

Kuntz, I. D. (1992). Structure-based strategies for drug design and discovery. Science 257, 1078-1082. doi: 10.1126/science.257.5073.1078

Lee, J. (2013). Cyclophilin A as a new therapeutic target for hepatitis C virusinduced hepatocellular carcinoma. Korean J. Physiol. Pharmacol. 17, 375-383. doi: 10.4196/kjpp.2013.17.5.375

Lee, J., and Kim, S. S. (2010). An overview of cyclophilins in human cancers. J. Int. Med. Res. 38, 1561-1574. doi: 10.1177/147323001003800501

Li, J., Chen, J., Zhang, L., Wang, F., Gui, C., Zhang, L., et al. (2006a). One novel quinoxaline derivative as a potent human cyclophilin A inhibitor shows highly inhibitory activity against mouse spleen cell proliferation. Bioorg. Med. Chem. 14, 5527-5534.

Li, J., Tan, J. Z., Chen, L. L., Zhang, J., Shen, X., Mei, C. L., et al. (2006b). Design, synthesis and antitumor evaluation of a new series of $\mathrm{N}$-substituted-thiourea derivatives. Acta Pharmacol. Sin. 27, 1259-1271.

Lucke, C., and Weiwad, M. (2011). Insights into immunophilin structure and function. Curr. Med. Chem. 18, 5333-5354. doi: 10.2174/092986711798194324

Miller, E. M., Xia, Q., Cella, M. E., Nenninger, A. W., Mruzik, M. N., BrillosMonia, K. A., et al. (2017). Voltammetric study of some 3-Aryl-quinoxaline2-carbonitrile 1,4-di-N-oxide derivatives with anti-tumor activities. Molecules 22:E1442. doi: 10.3390/molecules22091442

Muellenhoff, M. W., and Koo, J. Y. (2012). Cyclosporine and skin cancer: an international dermatologic perspective over 25 years of experience. A comprehensive review and pursuit to define safe use of cyclosporine in dermatology. J. Dermatolog. Treat. 23, 290-304. doi: 10.3109/09546634.2011. 590792 
Nigro, P., Pompilio, G., and Capogrossi, M. C. (2013). Cyclophilin A: a key player for human disease. Cell Death Dis. 4:e888. doi: 10.1038/cddis.2013.410

Nigro, P., Satoh, K., O'dell, M. R., Soe, N. N., Cui, Z., Mohan, A., et al. (2011). Cyclophilin A is an inflammatory mediator that promotes atherosclerosis in apolipoprotein E-deficient mice. J. Exp. Med. 208, 53-66. doi: 10.1084/jem. 20101174

Norman, K. G., Canter, J. A., Shi, M., Milne, G. L., Morrow, J. D., and Sligh, J. E. (2010). Cyclosporine A suppresses keratinocyte cell death through MPTP inhibition in a model for skin cancer in organ transplant recipients. Mitochondrion 10, 94-101. doi: 10.1016/j.mito.2009.10.001

Pap, T. (2005). Cyclophilins in rheumatoid arthritis-stepping into an undiscovered country? Clin. Immunol. 116, 199-201. doi: 10.1016/j.clim.2005. 06.013

Pereira, J. A., Pessoa, A. M., Cordeiro, M. N., Fernandes, R., Prudencio, C., Noronha, J. P., et al. (2015). Quinoxaline, its derivatives and applications: a state of the art review. Eur. J. Med. Chem. 97, 664-672. doi: 10.1016/j.ejmech.2014. 06.058

Qi, Z. Y., Hui, G. Z., Li, Y., Zhou, Z. X., Gu, S. H., Ying, K., et al. (2005). cDNA microarray in isolation of novel differentially expressed genes related to human glioma and clone of a novel full-length gene. Chin. Med. J. 118, 799-805.

Quarato, G., D’aprile, A., Gavillet, B., Vuagniaux, G., Moradpour, D., Capitanio, N., et al. (2012). The cyclophilin inhibitor alisporivir prevents hepatitis $C$ virusmediated mitochondrial dysfunction. Hepatology 55, 1333-1343. doi: 10.1002/ hep. 25514

Satoh, K., Nigro, P., Matoba, T., O’dell, M. R., Cui, Z., Shi, X., et al. (2009). Cyclophilin A enhances vascular oxidative stress and the development of angiotensin II-induced aortic aneurysms. Nat. Med. 15, 649-656. doi: 10.1038/ nm.1958

Sridevi, C., Balaji, K., and Naidu, A. (2011). Synthesis and pharmacological evaluation of some phenylpyrazolo indoquinoxaline derivatives. E-J. Chem. 8, 924-930. doi: 10.1155/2011/584817

Towers, G. J., Hatziioannou, T., Cowan, S., Goff, S. P., Luban, J., and Bieniasz, P. D. (2003). Cyclophilin A modulates the sensitivity of HIV-1 to host restriction factors. Nat. Med. 9, 1138-1143. doi: 10.1038/nm910

Walsh, C. T., Zydowsky, L. D., and Mckeon, F. D. (1992). Cyclosporin A, the cyclophilin class of peptidylprolyl isomerases, and blockade of T cell signal transduction. J. Biol. Chem. 267, 13115-13118.

Wang, G., Shen, J., Sun, J., Jiang, Z., Fan, J., Wang, H., et al. (2017). Cyclophilin A maintains glioma-initiating cell stemness by regulating Wnt/beta-catenin signaling. Clin. Cancer Res. 23, 6640-6649. doi: 10.1158/1078-0432.CCR-170774
Wang, P., and Heitman, J. (2005). The cyclophilins. Genome Biol. 6:226.

Weill, N., Therrien, E., Campagna-Slater, V., and Moitessier, N. (2014). Methods for docking small molecules to macromolecules: a user's perspective. 1 . The theory. Curr. Pharm. Des. 20, 3338-3359. doi: 10.2174/13816128113199990605

Weng, Q., Wang, D., Guo, P., Fang, L., Hu, Y., He, Q., et al. (2008). Q39, a novel synthetic Quinoxaline 1,4-Di-N-oxide compound with anti-cancer activity in hypoxia. Eur. J. Pharmacol. 581, 262-269. doi: 10.1016/j.ejphar.2007.12.006

Xue, C., Sowden, M., and Berk, B. C. (2017). Extracellular cyclophilin A, especially acetylated, causes pulmonary hypertension by stimulating endothelial apoptosis, redox stress, and inflammation. Arterioscler. Thromb. Vasc. Biol. 37, 1138-1146. doi: 10.1161/ATVBAHA.117.309212

Zarranz, B., Jaso, A., Aldana, I., and Monge, A. (2004). Synthesis and anticancer activity evaluation of new 2-alkylcarbonyl and 2-benzoyl-3-trifluoromethylquinoxaline 1,4-di-N-oxide derivatives. Bioorg. Med. Chem. 12, 3711-3721. doi: 10.1016/j.bmc.2004.04.013

Zhang, L., Qiu, B., Xiong, B., Li, X., Li, J., Wang, X., et al. (2007). Quinoxalinylurea derivatives as a novel class of JSP-1 inhibitors. Bioorg. Med. Chem. Lett. 17, 2118-2122. doi: 10.1016/j.bmcl.2007.01.094

Zhou, Z., Ying, K., Dai, J., Tang, R., Wang, W., Huang, Y., et al. (2001). Molecular cloning and characterization of a novel peptidylprolyl isomerase (cyclophilin)like gene (PPIL3) from human fetal brain. Cytogenet. Cell Genet. 92, 231-236. doi: $10.1159 / 000056909$

Ziaei, M., Ziaei, F., and Manzouri, B. (2016). Systemic cyclosporine and corneal transplantation. Int. Ophthalmol. 36, 139-146. doi: 10.1007/s10792-015-0137-8

Conflict of Interest Statement: Two patents related to this work have been granted in China: one for the compound 1,2-di(1H-pyrrol-2-yl)ethane-1,2-dione in 2011 (Patent No. ZL200910019140.2, inventors: XZ and CX) and the other for ZX-J-19j [N-(2,3-diphenylquinoxalin-6-yl)octanamide] in 2013 (Patent No. ZL201110178176.2, inventors: XZ and CX).

The other authors declare that the research was conducted in the absence of any commercial or financial relationships that could be construed as a potential conflict of interest.

Copyright $(2018$ Zhao, Xia, Wang, Wang, Xin, Yu and Liang. This is an open-access article distributed under the terms of the Creative Commons Attribution License (CC BY). The use, distribution or reproduction in other forums is permitted, provided the original author(s) and the copyright owner are credited and that the original publication in this journal is cited, in accordance with accepted academic practice. No use, distribution or reproduction is permitted which does not comply with these terms. 